\title{
Pyrolytic carbon: applications of its diamagnetism in metrology
}

\author{
Patrick Pinot ${ }^{*}$ and Zaccaria Silvestri \\ Laboratoire Commun de Métrologie LNE/CNAM, 1 rue Gaston Boissier, Paris 75724 Cedex 15, France
}

Received: 12 December 2018 / Accepted: 10 July 2019

\begin{abstract}
This paper presents some current or potential applications in metrology based on the diamagnetism of pyrolytic carbon $(\mathrm{PyC})$. The magnetic repulsion force acting between a piece of PyC subjected to a magnetic flux can be used as magnetic spring either to control the position of the sensitive sensor element or to detect changes of a physical quantity to be measured. The first part of this paper provides examples briefly described of devices based on the diamagnetism of $\mathrm{PyC}$ for measuring mechanical quantities. There are two main configurations for magnetic levitation: one, used for measuring acceleration or inclination for instance, is based on the levitation of a PyC tile above a magnet or set of magnets; In the other, used to measure forces caused for example by contact or buoyancy, it is the permanent magnet that is levitated at a stable height above a fixed $\mathrm{PyC}$ tile. The second part describes current work at the Laboratoire Commun de Métrologie (LCM-LNE/ CNAM) on the development of new laser power sensors using either diamagnetic force changes by photothermal excitation of electrons or diamagnetic torsion spring.
\end{abstract}

Keywords: Accelerometer / force meter / laser power / magnetic levitation / pyrolytic carbon / tiltmeter

\section{Introduction}

An object made from a diamagnetic material is repelled by a magnetic field. As a consequence of Earnshaw's theorem $[1,2]$, this property can be used to create a local minimum in the potential energy in magnetic fields and stabilize magnetic forces. Note that it took about 160 years from discovery of diamagnetism to the first practical demonstration of levitation [3]. Many natural substances (water, wood, carbon, etc.) or artificial ones (e.g. plastic) are weakly diamagnetic. The elements displaying the strongest diamagnetism at ambient temperature are the naturally occurring element bismuth and the man-made material pyrolytic carbon. In his $\mathrm{PhD}$ thesis, Pigot [4] showed how the diamagnetic properties of bismuth have been used for the design and fabrication of various microsensors and microactuators.

Pyrolytic carbon (PyC), developed in the 1960s, exists in several forms [5]. Highly oriented pyrolytic graphite (HOPG) composed of a stack along the $z$ direction of layers parallel to a $x-y$ plane, has interesting thermal, optical and electrical properties exploited for various applications, among them metrology. In addition, both its magnetic susceptibility and thermal conductivity are anisotropic. Since the late 1960s, its diamagnetic property has been exploited to develop devices using PyC levitation.

\footnotetext{
* Corresponding author: patrick.pinot@cnam.fr
}

This paper presents some features and benefits of $\mathrm{PyC}$ diamagnetism for applications in metrology in Section 2.

Then, the second part briefly describes some examples of current and potential metrological applications implementing the diamagnetism of $\mathrm{PyC}$ constituting the sensitive part of measuring devices such as accelerometer, tiltmeter, force meter and force-based sensor presented in Sections 3, 4 and 5 respectively.

Before the conclusion, Section 6 presents current work at the Laboratoire Commun de Métrologie (LCM-LNE/ CNAM) to develop laser power sensors based on the diamagnetism of $\mathrm{PyC}$.

\section{Pyrolytic carbon diamagnetism}

The PyC diamagnetism anisotropy is characterized by a magnetic susceptibility component $\chi_{z} \approx-4.5 \times 10^{-4}$ which greatly exceeds the components $\chi_{x}$ or $\chi_{y} \approx-0.85 \times 10^{-4}$. This strong directional diamagnetism can be used to stabilize, in a magnetic flux density, the free levitation of either a piece of $\mathrm{PyC}$ above a permanent magnet or a permanent magnet below a sheet of PyC.

In addition, the different magnetic stiffnesses generating restoring forces can be used for measuring external forces applied in translation along the $x, y$ or $z$-axes or in rotation around the $z$-axis.

Figure 1 shows an example of these two means of levitation. Note that stabilized levitation using $\mathrm{PyC}$ was 


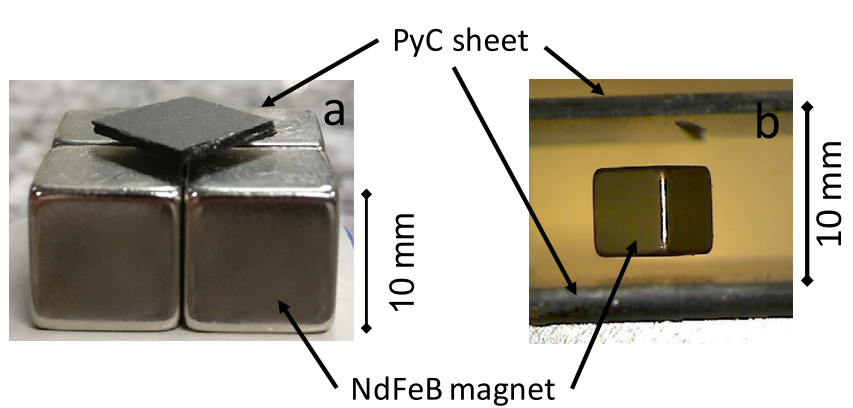

Fig. 1. Example of levitation at ambient temperature with no energy supplied: (a) square PyC tile levitated above an array of four cubic $\mathrm{NdFeB}$ magnets of side $10 \mathrm{~mm}$. (b) cubic $\mathrm{NdFeB}$ magnet of side $5 \mathrm{~mm}$ subjected to the attraction of a permanent magnet located above the device (not visible in the photograph) maintained in stabilized levitation between two PyC sheets.

patented for the first time [6] in 1971. In this patent concerning "diamagnetic levitation and/or stabilizing devices", the use of $\mathrm{PyC}$ levitation is clearly claimed as follows: "Another object of the invention is to provide diamagnetic levitation and/or stabilizing devices particularly adapted for the use of pyrolytic graphite diamagnetic members in relation to the magnetic fields of permanent magnets".

The three most remarkable advantages of the use of $\mathrm{PyC}$ diamagnetism for metrology are:

- first, PyC levitation in contrast to superconducting levitation [7] works at ambient temperature with no need for cryogenics nor indeed any energy input whatsoever if permanent magnets are used.

- secondly, the levitation of either a PyC plate or a permanent magnet as sensitive element means the measurement accuracy is limited neither by friction, nor parameters such as stiffness changes, wear thermal expansion and fatigue.

- thirdly, the low cost and the simple implementation of the base elements ( $\mathrm{PyC}$ plate and $\mathrm{NdFeB}$ magnets) which make it easy to develop a sensor based on $\mathrm{PyC}$ diamagnetism (levitation).

The stabilization of the levitation of a piece of $\mathrm{PyC}$ depends on the 3D magnetic field. Several authors [8-10] have studied the magnetic field distribution created by different arrangements of permanent magnets.

Since the 1960s, many applications for diamagnetic levitation appear to be promising [11] for sensing (e.g. accelerometers) and linkage devices of extremely low intrinsic friction (e.g. magnetic bearings or micro-rotors).

Note that most of sensors based on PyC diamagnetism presented here are not measurement standards (or etalons) but rather just sensors as defined by the international vocabulary of metrology [12], i.e. "element/s] of a measuring system that [are] directly affected by a phenomenon, body, or substance carrying a quantity to be measured', for which the physical stimulus can be heat, light, sound, pressure, magnetism, or a particular motion. Often, they have been developed for specific applications with constraints linked to measurement range, transducer design, implemented technology, environmental conditions, etc.

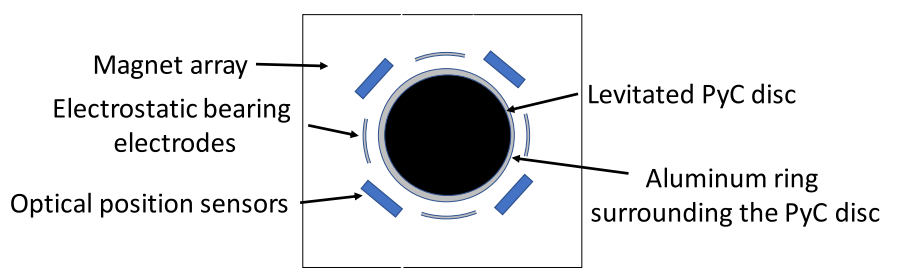

Fig. 2. Schematic diagram of the $2 \mathrm{D}$-accelerometer designed by Barrot et al. [21].

\section{Accelerometer}

\subsection{Principle}

Inertial sensors have an internal mass (seismic mass) in a spring system which any external acceleration causes to be displaced. They are used as accelerometers or gyroscopes to measure acceleration along a defined direction.

Micromachined high-resolution vibration sensors or accelerometers include capacitive [13-16], tunneling $[17,18]$, piezoresistive, optical [19], inertial MEMS [20], piezoelectric and ferroelectric sensors. There are also other types of industrial sensors based for instance on piezoresistivity (strain gauges), the (classical) Hall effect, mutual induction or eddy currents.

\subsection{Examples of device}

Several configurations of inertial sensors based on a $\mathrm{PyC}$ tile floating above a permanent magnet array have been designed. Their differences concern mainly the motion detection system.

Barrot et al. [21] have presented a novel force feedback acceleration sensor concept, featuring levitation of the inertial mass. Their device is a low cost 2D or 3Daccelerometer based on diamagnetic levitation and electrostatic actuation. The accuracy of this kind of device depends essentially on the electronics associated with the displacement detection of the $\mathrm{PyC}$ element. A PyC disc ( $28 \mathrm{~mm}$ diameter) surrounded by an aluminum ring which floats $250 \mu \mathrm{m}$ above a horizontal arrangement of permanent magnets constitutes the seismic mass $(1.68 \mathrm{~g})$.

Figure 2 shows the schematic diagram of a low-cost accelerometer in the 2D-configuration. The measuring range lies between $10^{-9} g$ and $1 g\left(g \approx 9.81 \mathrm{~m} \mathrm{~s}^{-2}\right.$ being the Earth's gravitational acceleration). For instance, the resolution of the $2 \mathrm{D}$ acceleration sensor is about $10^{-6} \mathrm{~g}$ $\left(\approx 10^{-5} \mathrm{~m} \mathrm{~s}^{-2}\right)$ at $0.5 \mathrm{~g}$. The sampling rate $(5 \mathrm{kHz}$ to $10 \mathrm{kHz})$ used for the position control of the levitated seismic mass is high compared to the frequencies of seismic signal $(0.1 \mathrm{~Hz}$ to $20 \mathrm{~Hz}$ ). One of the main potential applications of this sensor is seismic monitoring of civil engineering structures.

More recently, Andò et al. [22,23] have developed an acceleration sensor based on an inductive detection that can operate either in displacement or in acceleration mode. It is a low-cost differential inductive readout device based on an inertial sensor using a levitated $\mathrm{PyC}$ tile $\left(1.2 \mathrm{~cm}^{2}\right)$ suspended above four NdFeB magnets. The levitation mechanism allows low friction and high sensitivity. 


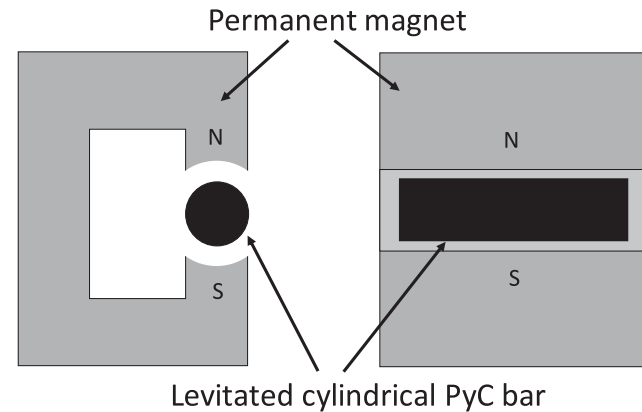

Fig. 3. Schematic diagram of the passive levitation system of the tiltmeter, designed by Simon et al. [24].

The sensitivity and the resolution of the sensor are $1.17 \mathrm{~V} /$ $\left(\mathrm{m} \mathrm{s}^{-2}\right)$ and $10^{-4} \mathrm{~m} \mathrm{~s}^{-2}$ in acceleration operating mode and $0.61 \mathrm{~V} / \mathrm{mm}$ and $1.9 \times 10^{-4} \mathrm{~mm}$ in displacement operating mode, respectively.

These capabilities can be compared with the limit of inrun bias stability for MEMS accelerometers, which is about $1.5 \times 10^{-4} \mathrm{~m} \mathrm{~s}^{-2}$ in acceleration operating mode, i.e. close to the resolution of $\mathrm{PyC}$-diamagnetism-based accelerometers, thus making the latter very competitive.

\section{Tiltmeter}

\subsection{Principle}

Tiltmeters are very sensitive inclinometers used to measure and record small deviations of a horizontal reference platform from orthogonality to the vector of local gravity. Equivalent to long-length stationary pendula, they are used for monitoring small changes on the ground (landslides such as rockfalls, mudflows, deformation of volcanoes, etc.) or in structures such as loading and foundation settlement.

\subsection{Example of device}

In the literature, we found only one example of an inclination sensor based on $\mathrm{PyC}$ levitation namely that described in 1968 by Simon et al. [24]. The tiltmeter is a horizontal pendulum type whose mass consists of a diamagnetic $\mathrm{PyC}$ rod suspended in the potential well of a suitably shaped magnetic field provided by a permanent magnet.

Figure 3 shows the schematic diagram of the passive $\mathrm{PyC}$ levitation system of the tiltmeter. The tiltmeter was designed for measurement of extremely small tilts of geophysical origin. The longitudinal movements of the $\mathrm{PyC}$ rod are used to measure inclination while its lateral movements are constrained by the strong stiffness provided by the magnetic field. The device can measure inclination in the range of $\pm 1 \mu \mathrm{rad}$ with a bandwith of $0.01 \mathrm{~Hz}$, but it is also very sensitive to vibrations.

Note that the resolution of the best tiltmeter is up to 1 nrad. This could be explained why there were no other development of tiltmeters using $\mathrm{PyC}$ levitation.

\section{Force sensor and force-based sensor}

\subsection{Principle}

A wide range of techniques is used to measure mechanical micro-forces. Such sensors are usually composed of an elastic structure on which the force being measured acts (cantilevers), and of a displacement or deformation sensor (strain gauge, capacitive, optical or piezoelectric detection, etc.). In addition, a balancing device generating an electrostatic, electromagnetic or piezoelectric force feedback can be added to improve accuracy.

All the sensors presented above use a PyC levitation configuration like that shown in Figure 1a, but in the case of force sensors, most of the examples found in the literature use levitation of a permanent magnet diamagnetically stabilized by pieces of $\mathrm{PyC}$ as shown in Figure $1 \mathrm{~b}$. Permanent magnets and coils allow one to maintain the permanent magnet in levitation subjected to the applied force to be measured.

There are several difficulties involved in measuring small contact forces and calibrating micro- and nano-force sensors. For measurement, one needs to determine and control the point of application and the alignment of the direction of force (a vector quantity). For calibration, one needs to understand the problems of coupling, interface and contact interaction (adhesion, friction) as well as the unwanted phenomena at the contact surface between the reference standard and the measurement device to be calibrated (sources of error).

There are also force-based sensors where a force is used for measuring other physical quantities. Typically, this force is generated either statically to balance a restoring force induced by the phenomenon, body or substance considered and linked to the quantity to be measured (buoyancy, stiffness, etc.) or dynamically to transfer energy to the system to determine the magnitude of the desired quantity (friction, thermal diffusion, etc.) from the system response.

\subsection{Examples of application}

\subsubsection{Contact force sensor}

At the FEMTO-ST institute in Besançon (France), Abadie et al. [25] have developed a low frequency micro- and nanoforce sensor based on a 10-cm long capillary tube used as levitating seismic mass. The force measurement range varies between $\pm 50 \mu \mathrm{N}$ and the resolution reached with a confocal chromatic sensor is about $5 \mathrm{nN}$. Figure 4 shows a schematic diagram of this force sensor using a levitating tube.

Another device has been developed by Cherry et al. [26] at the FEMTO-ST institute. Here, instead of a levitating tube, the seismic mass sensitive to external forces is a floating platform. The platform supports permanent magnets of which the magnetic forces interacting with external permanent magnets are stabilized by means of $\mathrm{PyC}$ plates. Three confocal sensors are used to measure the displacements of the platform. The measurement range varies between $\pm 50 \mu \mathrm{N}$ while the resolution can reach less than $10 \mathrm{nN}$ if an anti-vibration table is used. 


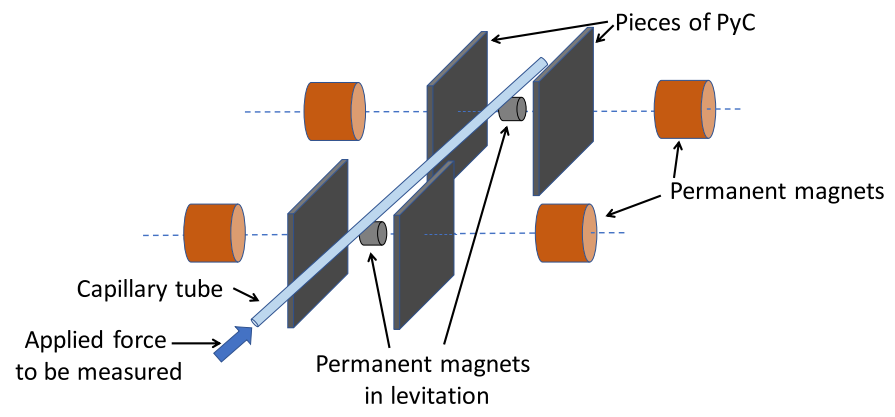

Fig. 4. Schematic diagram of the principle of force sensor using stabilized levitation of permanent magnets designed at the FEMTO-ST institute in Besançon (France).

In another example, Li et al. [27] have developed a diamagnetic lateral force calibrator composed of four $\mathrm{NdFeB}$ magnets and a diamagnetic pyrolytic graphite sheet to calibrate directly an atomic force microscope cantilever-tip or -bead assemblies. This sensor provides both the lateral force and the crosstalk lateral force constants with an accuracy on the order of $0.1 \%$. The spring constant of about $10 \mathrm{pN} / \mathrm{nm}$ is measured with $10^{-4}$ relative accuracy and is used for calibrating the AFM lateral constants. The location uncertainty of the shear centre of the cantilever constitutes the main difficulty of the measurement method.

All these devices based on PyC diamagnetic present interesting features. For comparison in terms of resolution, Fahlbush and Fatikow [28] have tested semiconductor strain gauges attached to a microgripper used as a self-sensing cantilever for atomic force microscopes. The resolution is about $2 \mathrm{nN}$ in the range of a few micronewtons. Kim et al. [29] have presented a monolithic MEMS-based microgripper to manipulate biological cells. The device has an integrated force feedback based on capacitive force sensor with a gripping force resolution of about $20 \mathrm{nN}$ in the range of a few tens of micronewtons.

Whatever the case, calibration of small force sensors is particularly complicated and lacks real traceability. However, although the calibration is for the sub-piconewton range, the work of Melcher et al. [30] can be mentioned. The authors have developed an ultrasensitive optomechanical sensor with a stiffness of $4 \mu \mathrm{N} / \mathrm{nm}$, designed to improve the accuracy and precision of force measurements with atomic force microscopy. Its self-calibration is realized through using the radiation pressure from light incident on the cantilever surface. The force resolution of this device is estimated to be $14 \mathrm{fN}$ in the range from zero to a few hundreds of a femtonewton.

\subsubsection{Viscosity and density sensor}

Clara et al. [31] have investigated the feasibility of using a "floater" magnet (a permanent magnet in levitation about $0.6 \mathrm{~mm}$ above a piece of $\mathrm{PyC}$ ) immersed in a liquid to measure its density and viscosity. Figure 5 shows the principle of the set-up developed at the Johannes Kepler University in Linz (Austria).
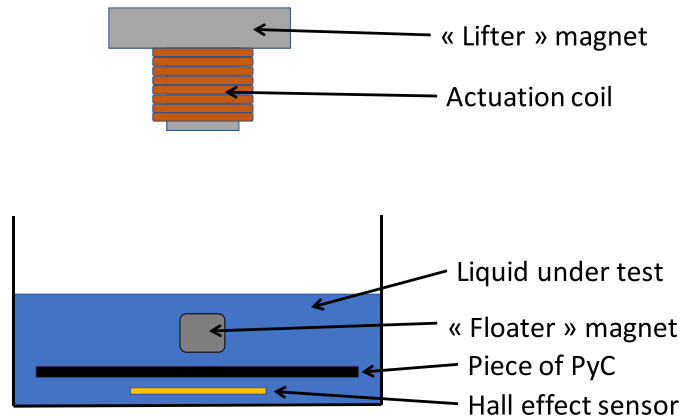

Fig. 5. Schematic diagram of the set-up designed by Clara et al. [31].

The density of the fluid can be determined by balancing the buoyancy force and the magnetic force acting on the "floater" magnet to keep its levitation height constant in the liquid under test. The "floater" magnet is levitated using a "lifter" magnet (permanent magnet). The height of the "floater" magnet is measured over the vertical magnetic flux density by a Hall effect sensor placed beneath the pyrolytic carbon. The position of the "lifter" magnet is adjusted to keep the height of the "floater" magnet constant. There is a relation between the position of the "lifter" magnet and the buoyancy force which is related to the density of the fluid. This method is applicable for a large range of densities from below $1 \mathrm{~kg} / \mathrm{m}^{3}$ (approximately that of air) to $1300 \mathrm{~kg} / \mathrm{m}^{3}$ (1.3 times that of water), but the relative error depends on the levitation height. For instance, the relative error in the density measurement lies between $0.1 \%$ and $1 \%$ for a levitation height of $0.75 \mathrm{~mm}$. The sensitivity of the experimental set-up is about $4 \mu \mathrm{m} /\left(\mathrm{kg} \mathrm{m}^{-3}\right)$ and the resolution in height measurement is about $0.01 \mathrm{~mm}$.

For viscosity measurements, an AC-driven coil is added to the "lifter" magnet. The AC-component superposes an alternating magnetic force which induces oscillations of the "floater" magnet. The resonance frequency for the set-up is around $6 \mathrm{~Hz}$. The damping of the resonance is related to the viscosity of the fluid. The quality factor falls with increasing dynamic viscosity. The best results are obtained for low viscosities in the range below $20 \mathrm{mPa}$. No estimate of measurement uncertainty is provided in the paper.

Note that the accuracy of this device is not very high compared with classical methods used at any National Metrology Institute. For instance, the determination of the density of a liquid by means of the pycnometric method is provided with relative uncertainty of about $0.05 \%$ and the relative uncertainty for measurement of dynamic viscosity in the range below $20 \mathrm{mPa} \mathrm{s}$ is about $0.5 \%$.

\section{Laser power meter}

\subsection{Photo-thermal excitation}

Laser power meters are mainly based on either thermal measurement techniques via the Stefan-Boltzmann law (thermopile sensors) or the photon-electron interaction (photodiode sensors). The LCM/LNE-CNAM is currently 
developing three experimental configurations based on a repulsive force acting on a piece of $\mathrm{PyC}$ in a magnetic induction generated by a magnet array to measure laser power in an intermediate range from a few hundred of milliwatts to a few watts.

The first two are based on the change of $\mathrm{PyC}$ diamagnetism due to photo-thermal excitation of electrons by laser radiation. The third uses 3D-stabilisation of a levitated $\mathrm{PyC}$ disc as a passive magnetic spring to detect the radiation pressure of a laser beam. Up to now, most of our experimental measurements have been carried out for the power range $100 \mathrm{~mW}$ to $1 \mathrm{~W}$ at a wavelength of $1064 \mathrm{~nm}$.

The experimental setup of the first configuration [32] similar to that of Figure 1a is very simple. In this case, the repelling diamagnetic force is modified by the laser beam irradiating with an incidence angle perpendicular to the $\mathrm{PyC}$ surface that involves the levitation height change which is measured by means of an optical position sensor with a resolution of $10 \mathrm{~nm}$. There is a quasi-linear relation between the optical power change and the levitation height change. The photo-response sensitivity of this laser power meter is about $100 \mathrm{~mW} \mathrm{\mu m}^{-1}$ and its discrimination threshold is about $3 \mathrm{~mW}$. The main environmental quantities influencing the measurement are temperature and the background noise due to vibrations and movements of air. Its relative uncertainty for optical power measurement between 0.4 and $1 \mathrm{~W}$ at $1064 \mathrm{~nm}$ is less than $10 \%$. The accuracy of this device is not competitive with that of photodiode or thermopile sensors where the laser power measurement uncertainty is close to $1 \%$ just after calibration. Figure 6 a shows the photograph of a set-up and Figure $6 \mathrm{~b}$ gives an example of response in terms of displacement measured by the optical position sensor when the $\mathrm{PyC}$ surface is illuminated by the beam of the laser under test.

The second configuration [33] does not use levitation, but instead the magnetic interaction force between a permanent magnet fixed above a PyC tile placed on the balance pan of scales. The optical power is proportional to the mass change which corresponds to a magnetic repulsion force change acting on the $\mathrm{PyC}$ tile irradiated by a laser beam. The photo-response sensitivity of this laser power sensor is about $100 \mathrm{~mW} \mathrm{mg}^{-1}$ and its discrimination threshold is about $1 \mathrm{~mW}$. The main quantities influencing the measurement are wavelength, spot position on the $\mathrm{PyC}$ surface and power density of the laser under test. The relative uncertainty of the optical power measurement at $1064 \mathrm{~nm}$ decreases from $2 \%$ at $0.1 \mathrm{~W}$ to $0.8 \%$ at $1 \mathrm{~W}$. This measurement uncertainty is quite like that of photodiode and thermopile sensors. However, the setup using accurate scales is not simple to use. Figure 7a shows a photograph of the set-up for the second experimental configuration. An example of response in terms of mass after $60 \mathrm{~s}$ irradiation for three different laser powers at $1064 \mathrm{~nm}$ is given in Figure $7 \mathrm{~b}$.

\subsection{Diamagnetic spring}

The third configuration [34] when its physical parameters (moment of inertia, natural frequency, logarithmic a
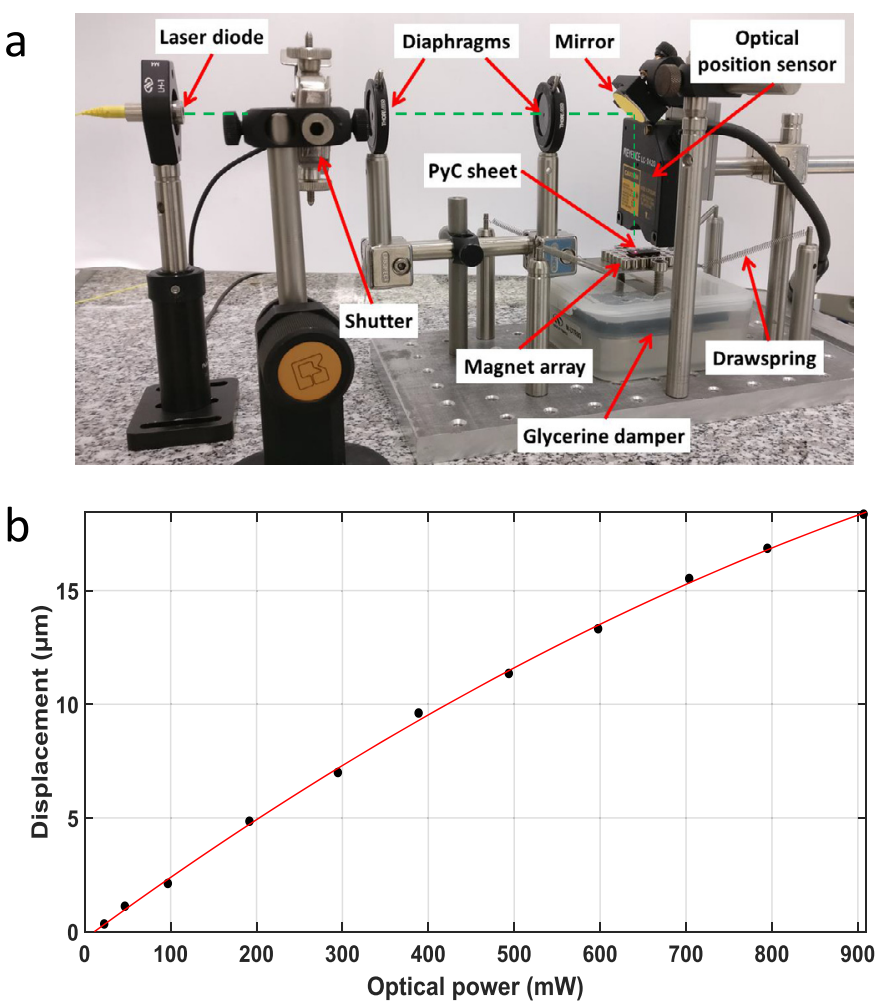

Fig. 6. First configuration: Levitation height change of the $\mathrm{PyC}$ piece levitated above the magnet when its surface is irradiated by a laser beam (green dashed line). (a) Photograph of a setup with a vibration absorber where the magnet array is guided by way of helical springs and a metal block fixed to the magnet array is immersed in glycerine. (b) Response in terms of displacement after $60 \mathrm{~s}$ irradiation at $532 \mathrm{~nm}$ for $20 \mathrm{~mW}$ and at $1064 \mathrm{~nm}$ above $20 \mathrm{~mW}$.

decrement, etc.) are determined could provide an absolute power measurement without calibration unlike to the two configurations presented in Section 6.1 which need to be calibrated by using laser sources and optical power meters as a reference. It uses the diamagnetic spring composed of a $\mathrm{PyC}$ piece levitated above a permanent magnet array. In this case, the $\mathrm{PyC}$ piece itself must not be irradiated by the laser beam under test. Figure 8a shows a photograph of an experimental setup of this inexpensive, sensitive, passive laser power meter. The measurement of radiation force is based on a torsional spring-mass-damper system consisting of a PyC disc $(\varnothing=20 \mathrm{~mm})$ levitated above a $\mathrm{NdFeB}$ magnet array.

The nine-magnet array provides a magnetic field distribution with a $2 \mathrm{D}$ periodicity of the vertical component of the magnetic flux $B_{z}$. The levitation force of the $\mathrm{PyC}$ disc is due to the interaction of the $\mathrm{PyC}$ diamagnetism in $B_{z}$ and its $2 \mathrm{D}$ periodicity allows stabilizing the $\mathrm{PyC}$ disc over the center of the magnet array. To provide a steady angular position and thus a torsional restoring force, three holes $(\varnothing=1.5 \mathrm{~mm})$ were drilled at the vertices of an equilateral triangle in the $\mathrm{PyC}$ disc. These holes are used to locate three polyamide spheres.

The measurement method is dynamic consisting in analyzing the response of the system to a rising or a failing 


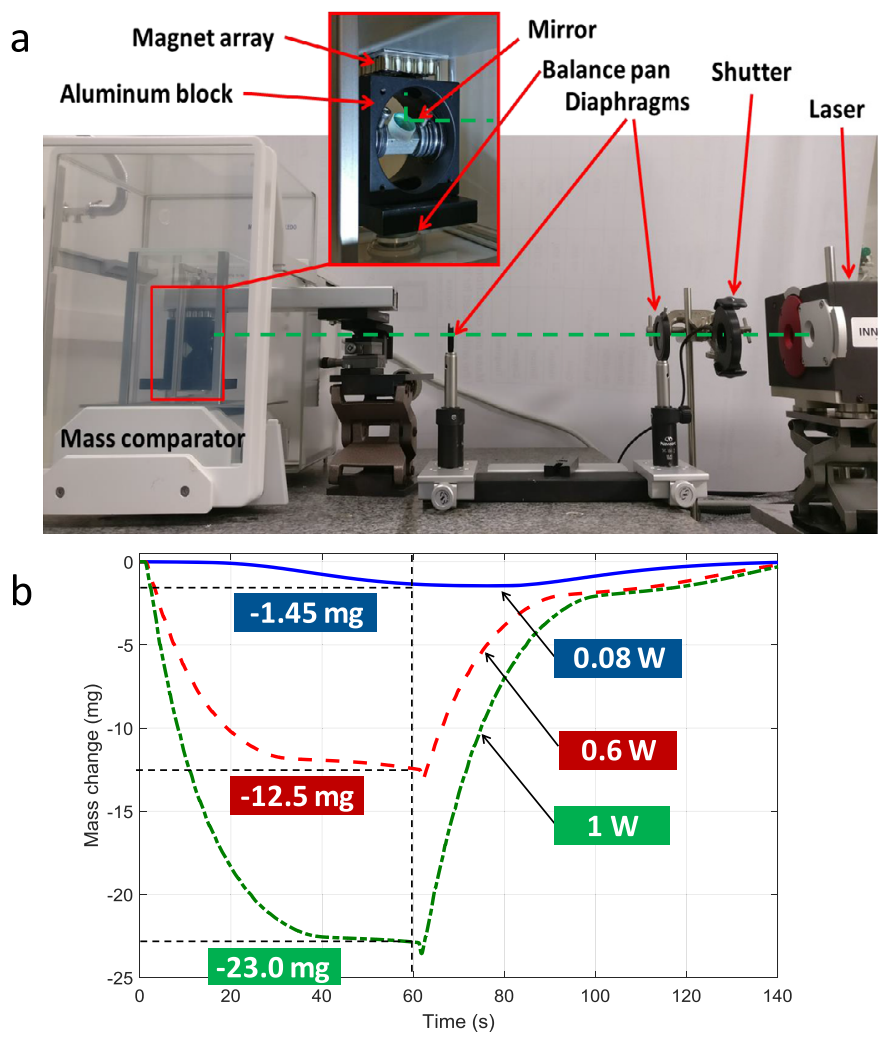

Fig. 7. Second configuration: Mass change due to the repulsion force change between the $\mathrm{PyC}$ piece placed on a balance pan and the magnet array held above it when its surface is irradiated by a laser beam (green dashed line). (a) Photograph of a setup using a mass comparator Mettler-Toledo AX206. The detailed view in box shows the aluminium block with the mirror placed on the balance pan and the magnet array fixed above the $\mathrm{PyC}$ tile (the $\mathrm{PyC}$ tile glued on the aluminium block is not visible on the photograph). (b) Example of response in terms of mass after $60 \mathrm{~s}$ irradiation for three different laser powers at $1064 \mathrm{~nm}$.

edge of a periodically interrupted laser beam. In this way, the angular response of the $\mathrm{PyC}$ disc to laser power remains linear over the measurement range. Figure $8 \mathrm{~b}$ shows an example of response.

The first experimental results give a relative expanded uncertainty for measurement of optical power of $10 \%$ at $450 \mathrm{~mW}$ and $5 \%$ at $1 \mathrm{~W}$ and suggest an accuracy of $1 \%$ could be achieved for $5 \mathrm{~W}$. Amongst the main sources of uncertainty are the moment of inertia of the levitated system and the measurements of the PSD voltage and the pseudo-period of the damped oscillation.

However, several parts of this device could be improved to reduce the laser power measurement uncertainty. These include:

- the techniques used for dimensional measurements to calculate the optical lever arms (from mirror for detection to PSD sensor and from mirror for laser under test to the rotation centre of the levitated part) and the moment of inertia of the levitated elements (PyC disc, polyamide spheres, cover glass, right-angle prism mirror);

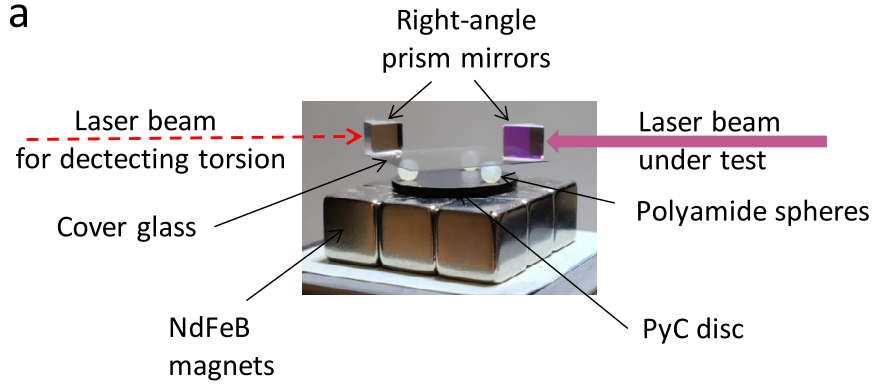

b

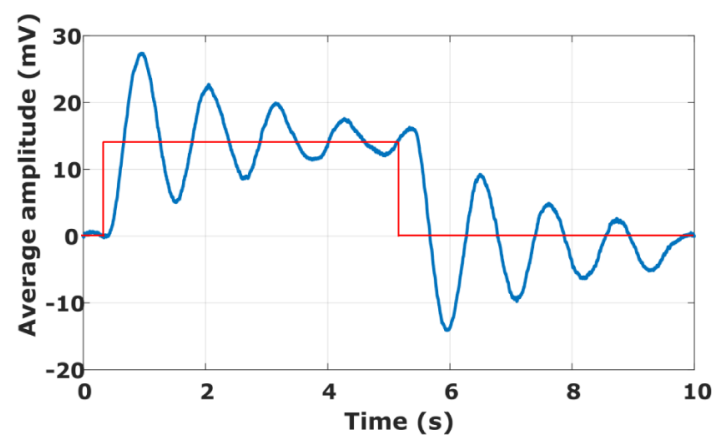

Fig. 8. Third configuration: Radiation force meter based on a torsional spring-mass-damper system consisting of a small pyrolytic carbon disk levitated above a permanent magnet array. (a) Photograph of the passive sensitive system showing: - arrangements of nine cubic NdFeB magnets $(10 \mathrm{~mm} \times 10 \mathrm{~mm}$ $\times 10 \mathrm{~mm}$ ); -levitated PyC disc (diameter: $20 \mathrm{~mm}$ ); - three polyamide spheres (diameter: $3 \mathrm{~mm}$ ); - square cover glass $(18 \mathrm{~mm} \times 18 \mathrm{~mm})$; - two right-angle prism mirrors (one used for measuring the angular deflection, the other illuminated by the laser beam under test). (b) Example of response in terms of voltage given by a position sensitive detector measuring the angular deflection displacement of the detection laser beam to a $1 \mathrm{~W}$ radiation step for $5 \mathrm{~s}$ (red square signal) provided by the laser under test.

- the detection sensitivity by using a longer cover glass to increase the optical lever arm and by using a new PSD sensor (more linear and more sensitive);

- the response linearity not only by using the new PSD but also by placing the right-angle prism mirror used for deflecting the detection laser beam at the rotation centre (it is not the case in Fig. 8a).

In this way, a relative expanded uncertainty of $1 \%$ for a smaller optical power than $1 \mathrm{~W}$ should be achievable, making this device potentially a very competitive measurement standard.

The main constraint for these three configurations is that they can work only when the PyC piece is perfectly adjusted in the horizontal plane. Consequently, the direction of the laser beam under test must be adjusted too with respect to the $\mathrm{PyC}$ piece.

\section{Discussion and conclusion}

The anisotropic diamagnetism of $\mathrm{PyC}$ allows one to use stabilized levitation at ambient temperature with no need 
for superconductors and their associated energy input and thus at very low cost. This remarkable property can be used for linkage devices and sensing. Some examples of applications show it could be interesting for metrology to use the diamagnetism of $\mathrm{PyC}$ as the sensitive element of various gauges.

All these sensors are used for measuring mechanical quantities such as acceleration, force, inclination, density or viscosity. Most of them are composed of an inexpensive primary sensor using magnets and $\mathrm{PyC}$ tiles combined with a secondary sensor. The PyC-based sensor is directly sensitive to the physical quantity to be measured and in general its response to a stimulus is a displacement (translation or rotation). The secondary sensor is often an optical sensor with a transducer delivering an electrical signal used to measure either a linear displacement or an angular deflection.

As with most sensors, environmental conditions (temperature, vibration, draughts, etc.) can have a more or less great effect on magnetic levitation and thus on the measurement results obtained with magnetic levitation sensors. Their influence must be reduced to obtain the best measurement accuracy. It is usually necessary to add sensors to measure quantities affecting accuracy in order to apply corrections to the measurement signal. Feedback systems can be also used to improve the accuracy of the PyC-based sensors. This is the case for instance for the accelerometer presented above, but also for the laser power sensor using a balance which has an internal feedback system maintaining the balance pan at a constant height.

Our recent and ongoing work at the LCM-LNE/CNAM has demonstrated the feasibility of measuring laser powers using $\mathrm{PyC}$ diamagnetism. Two effects can be used for this: - Photo-thermal excitation of electrons induced by direct irradiation of the levitated $\mathrm{PyC}$ sheet, in this case the magnetic interaction force change which can be measured in terms of either levitation height change or mass change is related to the optical power of the laser beam.

- A diamagnetic spring related to the stiffness of the levitation force, in this case the levitated $\mathrm{PyC}$ sheet is used as a rotational spring pendulum for which the angular deflection is proportional to radiation pressure provided by a laser beam.

To conclude, this paper has shown that the main advantage of the $\mathrm{PyC}$ diamagnetism is to provide a lowcost element sensitive to motion in terms of inertia or inclination and force due to restoring magnetic force. To exploit its sensitivity, it must be associated with a suitable displacement detection and possibly a feedback system too.

Most of examples of PyC-based sensors presented in this paper remain laboratory sensors, not yet implemented in a routine way in metrology. Of course, while $\mathrm{PyC}$ diamagnetism is used in many applications to stabilize position, steer movement or avoid friction, it is rarely used as the sensitive element for measuring a physical quantity.

There might be other potential applications using $\mathrm{PyC}$ levitation for measurement and control for a wide range of instruments such as flowmeters, gravity meters, magnetometers or micrometers. In addition, as suggested by
Küstler [35], recent developments in the new technology of printing magnet field domains could offer new opportunities of applications for metrology.

The authors thank Dr Mark Plimmer for his kind reading of the manuscript.

\section{References}

1. S. Earnshaw, Trans. Camb. Phil. Soc. 7, 97-112 (1842)

2. R. Bassini, Meccanica 41, 375-389 (2006)

3. G. Küstler, Revue Roumaine des Sciences Techniques - Serie Électrotechnique et Énergétique 52, 265-282 (2007)

4. C. Pigot, Ph.D. thesis, Institut National Polytechnique de Grenoble, 2008

5. L.C.F. Blackman, A.R. Ubbelohde, Proc. Roy. Soc. Lond. A 266, 20-32 (1962)

6. US patent $3,597,022$, submitted in 1969 and corrected in 1971, U S3597022.

7. V. Arkadiev, Nature 160, 330 (1947)

8. F. Barrot, Ph.D. thesis, École Polytechnique Fédérale de Lausanne, 2008

9. G. De Pasquale, S. Iamoni, A. Somà, Int. J. Mech. Sci. 68, 56-66 (2013)

10. M.D. Simon, L.O. Heflinger, A.K. Geim, Am. J. Phys. 69, 702 (2001)

11. R.D. Waldron, Rev. Sci. Instrum. 37, 29-35 (1966)

12. International vocabulary of metrology - Basic and general concepts and associated terms (VIM) JCGM 200:2012, 3rd edition, BIPM

13. W. Henrion, L. DiSanza, M. Ip, S. Terry, H. Jerman, in Tech. Dig. 1990 Solid-State Sensor and Actuator Workshop, Hilton Head Island, SC, June 4-7, 1990, pp. 153-157

14. K. Bult, A. Burstein, D. Chang, in Proc. 1996 Hilton Head Solid State Sensor and Actuator Conf., 1996, 205-210.

15. M.A. Lemkin, M.A. Ortiz, N. Wongkomet, B.E. Boser, J.H. Smith, in Tech. Dig. 9th Int. Conf. Solid-State Sensors and Actuators (Transducers'97), Chicago, IL, June 1997, 1997, $1185-1188$.

16. K.H.-L. Chau, S.R. Lewis, Y. Zhao, R.T. Howe, S.F. Bart, R.G. Marcheselli, Sens. Actuat. A 54, 472-476 (1996)

17. C.H. Liu, J.D. Grade, A.M. Barzilai, K.K. Reynolds, A. Partridge, J.J.K. Rockstad, T.W. Kenney, in Transducers '97 Tech. Dig., 471-472, paper 2B3.07, 1997

18. H.K. Rockland, T.W. Kenny, P. Kelley, T. Gabrielson, in Proc. Acoustic Particle Velocity Sensors: Design, Performance, and Applications, 1996, 57-68

19. T.K. Gangopadhyay, Sens. Actuat. A 113, 20-38 (2004)

20. J. Bernstein, R. Miller, W. Kelley, P. Ward, J. Microelectr. Syst. 8, 433-438 (1999)

21. F. Barrot, J. Sandtner, H. Bleuler, in IUTAM Symposium on Vibration Control of Nonlinear Mechanisms and Structures, edited by H. Ulbrich, W. Günthner. Solid Mechanics and its Applications. (Springer, Dordrecht, 2005), Vol. 130

22. B. Andò, S. Baglio, V. Marletta, A. Valastro, A. Pistorio, C. Trigona, in 2017 IEEE International Instrumentation and Measurement Technology Conference (I2MTC), 2017

23. B. Andò, S. Baglio, V. Marletta, V. Valastro, IEEE Trans. Instr. Meas. 67, 1238-1245 (2018)

24. I. Simon, A.G. Emslie, P.F. Strong, R.K. McConnell, Jr, Rev. Sci. Instrum. 39, 1666-1671 (1968) 
25. J. Abadie, E. Piat, S. Oster, M. Boukallel, Sens. Actuat. A 173, 227-237 (2012)

26. A. Cherry, J. Abadie, E. Piat, Sens. Actuat. A 169, 27-36 (2011)

27. Q. Li, K.-S. Kim, A. Rydberg, Rev. Sci. Instrum. 77, 065105 (2006)

28. S. Fahlbush, S. Fatikow, in Proc. IEEE Int. Conf. on Robotics and Automation, 2001, 3435-3440

29. K. Kim, X. Liu, Y. Zhang, Y. Sun, J. Micromech. Microeng. 18, 213-550138 (2008)
30. J. Melcher, J. Stirling, F. Guzmán Cervantes, J.R. Pratt, G.A. Shaw, Appl. Phys. Lett. 105, 233109 (2014)

31. S. Clara, H. Antlinger, W. Hilber, B. Jakoby, IEEE Sensors J. 15, 1937-1944 (2015)

32. P. Pinot, Z. Silvestri, Rev. Sci. Instrum. 88, 085003 (2017)

33. P. Pinot, Z. Silvestri, Measur. Sci. Technol. 29, 015103 (2018)

34. P. Pinot, Z. Silvestri, Measurement 131, 109-119 (2019)

35. G. Küstler, Electr. Lett. 50, 1289-1290 (2014)

Cite this article as: Patrick Pinot, Zaccaria Silvestri, Pyrolytic carbon: applications of its diamagnetism in metrology, Int. J. Metrol. Qual. Eng. 10, 7 (2019) 